\title{
Innovative Robot Design for Cleaning Solar Panels
}

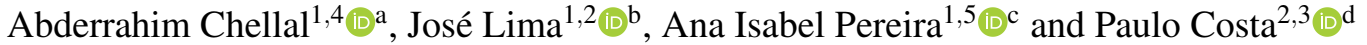 \\ ${ }^{1}$ Research Centre of Digitalization and Intelligent Robotics CeDRI, Instituto Politécnico de Bragança, Bragança, Portugal \\ ${ }^{2}$ Robotics and Intelligent Systems Research Group, INESC TEC, Porto, Portugal \\ ${ }^{3}$ Faculty of Engineering of the University of Porto, DEEC, Porto, Portugal \\ ${ }^{4}$ Higher School in Applied Sciences of Tlemcen, Tlemcen, Algeria \\ ${ }^{5}$ ALGORITMI Center, University of Minho, Braga, Portugal
}

Keywords: Robotics, Automation and Mechatronics, Solar Energy, Data Analytics and Simulation, Digital Image Processing, Efficiency Increase.

\begin{abstract}
The accumulation of dust particles on the solar panels decrease the total amount of solar energy received by the Photovoltaic panel (PV) and, it has also been proven that the increase of temperature reduces the overall efficiency. These effects have been studied by different researchers based on collected data comparison. As both of these parameters have a negative effect on the efficiency of the solar panel, it is essential to keep them clean and at low temperatures. The use of technologies, such as robots, is an effective way of carrying out repetitive tasks at low cost and in a short time. This work consists of developing a robot capable of cleaning and cooling the solar panels, based on images acquired through a camera positioned directly towards the panels, thus maintaining periodic cleaning in order to increase its efficiency. The preliminary results demonstrate the possibility of using this approach for the accomplishment of this task.
\end{abstract}

\section{$1-$ INTRODUCTION}

Renewable energy plays an important role in the energy sector by replacing limited resources such as oil and coal with abundant natural resources. One of the most popular of these renewables is solar energy. Solar energy is radiant energy emitted by the sun and harnessed by photovoltaic panels. It offers various advantages over other available renewable energy sources, but suffers from several drawbacks.

The photovoltaic module is a set of cells connected to each other in series and/or parallel, which produces a current and a voltage when exposed to the sun. (Campana et al., 2013). Environmental factors such as temperature, wind and rain greatly influence the reliability and efficiency of solar panels and represent one of the major problems of this technology. It has also been shown that the accumulation of dust particles significantly reduces the overall efficiency, especially in desert areas, but not only.

\footnotetext{
a(i) https://orcid.org/0000-0002-9190-6865

b (D) https://orcid.org/0000-0001-7902-1207

c (iD https://orcid.org/0000-0003-3803-2043

d(D) https://orcid.org/0000-0002-4846-271X
}

During long periods without rain, such as the summer, dust accumulation can lead to daily losses of over 20\%. (Zorrilla-Casanova et al., 2011), these losses lead to a substantial decrease in the overall efficiency of photovoltaic systems.

Figure 1 shows dirty and clean solar panels.

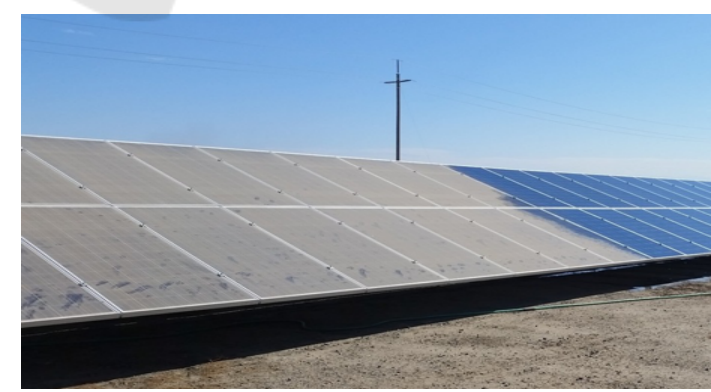

Figure 1: Dirty and clean Solar panels (JKB Energy, 2018).

The effect of dust accumulation on solar cells varies with location and has been studied by a number of researchers. In (Christian et al., 2015) It is revealed that a $20 \%$ drop in efficiency was observed on photovoltaic panels installed in Freiburg, Germany, within 5 months of heavy soiling of the glass surface. In 
the Algerian Sahara desert, it was found that the efficiency of solar panels decreased by $32 \%$ after only 6 months without any cleaning operation. (Mostefaoui et al., 2019). In these cases, regular cleaning of the modules is necessary. It is strongly recommended that, when weather conditions are not extreme, the photovoltaic panels are cleaned 3 to 4 times a year, and this number should increase during dry periods. Usually this is done regularly by human operators, but it is time-consuming and, more importantly, generates additional operating costs ranging from $€ 1000$ to $€ 4500 / \mathrm{MW}$ depending on the region. (Stridh, 2012).

The objective of this paper is to propose a methodology, based on simulation, that describes an efficient and economical way to perform this repetitive, timeconsuming and costly task by using robots in an innovative way, through the use of a camera, giving the robot autonomy and greater work efficiency. This document is divided into seven sections. The first section presents the overview of the work. The second section promotes the work already done in this area, followed in section 3 , by a brief description of the overall system architecture. The environment and the simulation of the model are presented in section 4 where the robot simulation software is described. The fifth section introduces in general the algorithms applied in the different software, while the sixth section highlights the results obtained, and finally ends with a conclusion and future work.

\section{RELATED WORK}

Some reported automatic systems were proposed in the literature to solve the dust accumulation issue through robots. In (Ronnaronglit and Maneerat, 2019) a freely moving robot is suggested: it can go in any direction and is able to climb a maximum of $20^{\circ}$ slope inclination. In (Anderson et al., 2010), a two part rail guided robot is designed, it consist of a " Drive System" that moves horizontally along the top and bottom edges of the solar panel, and a "Cleaning Head" that is driven vertically by cables.

In (Aravind et al., 2014) the proposed system is made up of two sub-system, a robot cleaner and an automated carrier cart, while the robot clean the PV panel surface, the carries moves along side.

In (Sundaram et al., 2019) The focus was on the concept of a robot that could be installed in any solar farm, easy to use and particularly easy to transport. A market survey and patent analysis on the use of Solar cleaning robots is presented in (Grando et al., 2019), which offers a general view of different approach and technologies involved and commercialized.
In (Hashim et al., 2019) The proposed strategy allows both monitoring of solar irradiation and cleaning of the photovoltaic surface when necessary through a camera mounted directly on the robot, under the control of a mobile application that also collects the data. Another cleaning strategy using a camera was also proposed in (Li et al., 2016), where a mapping framework was developed using a Kinect V2 as an RGB-D sensor.

\section{SYSTEM ARCHITECTURE}

The system architecture is based on two components, the SimTwo software, where the robot is fully simulated, and a processing application developed on Lazarus Software, a cross-platform IDE for application development. These two platforms communicate with each other using the User Datagram Protocol (UDP). Camera image is compressed on jpeg format and sent by port 9808 from SimTwo to the application whereas on the opposite way, the SimTwo will receive commands on port 9898 to control the motors.

The use of SimTwo is justified by the need to perform the simulation of the robot while Lazarus is used to develop an application capable of processing the image acquired by the camera in the SimTwo software and controlling the robot. The application consists of the analysis of the image, the determination of the location and edges of the solar panels and the exact coordinates of the dust on them, this will be further discussed afterwards.

Figure 2 shows the main architecture of the proposed system.

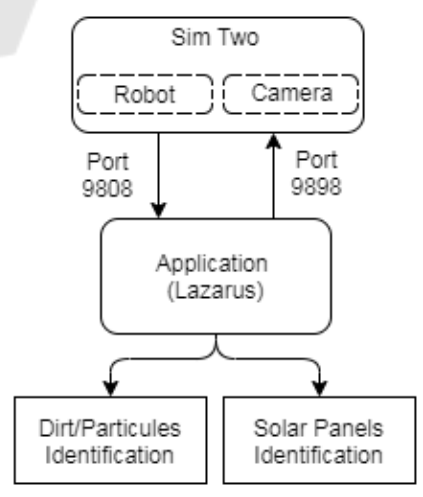

Figure 2: System Architecture.

The same approach is to be used in a real scenario, replacing the SimTwo simulation environment block with the real robot and camera. In this case, the camera will send data directly to the application, and the robot will communicate with the application via $\mathrm{WiFi}$ 
or another network, depending on the infrastructure.

\section{ENVIRONMENT AND MODEL SIMULATION}

SimTwo is a realistic mobile robot simulation software with a multiple window interface that can support several types of robots with dynamic capability (Gonçalves et al., 2013) and it is useful for development and testing purposes. It is strongly recommended to create the robot model with dimensions, positions and part masses very close to the real parts, since the dynamics will be calculated based on these characteristics and it is desired that it behaves like a real situation. Figure 3 represents an overview of the different interface of the software.

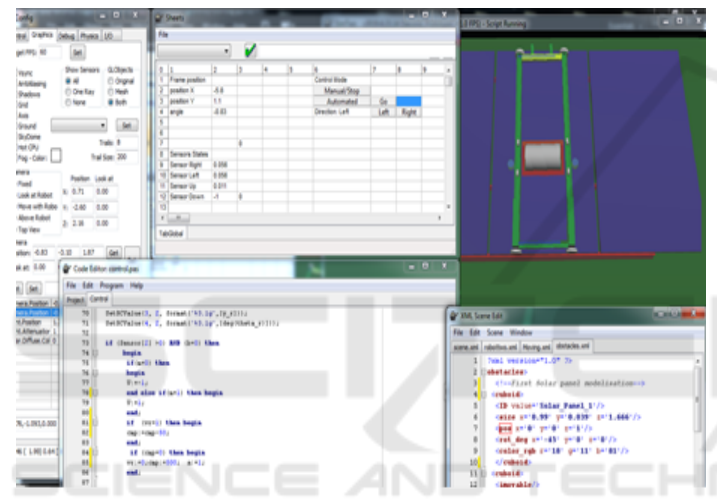

Figure 3: SimTwo software - General view.

In this section, it will be discussed how the development of the scene environment on the software was carried out, firstly by modelling the solar panels presented in the CeDRI facilities, and next, by modeling the main parts of the cleaning robots, which are the frame and the mobile robot. The robot's look and behavior are defined in several XML format files and are implemented in the "scene editor" window. The main scene file (scene.xml) defines the different sub-robot parts incorporated in the main robot and their specific construction file. The robot parts are defined by several solids (cuboid and cylinder) connected through joints and articulations (slider and hinge). These joints are parts of the shell elements since they are solids without mass and do not modify the robot's physical properties, but are an essential part of the collision simulation (Costa et al., 2011). The movement occurs through a guide rails, called drive frame, fixed in the edges of the solar panel, allowing a fluid movement of the mobile robot and giving it the possibility of mounting a steeply sloping panel. The drive frame should be manufactured using aluminium al- loy, offering moderate strength, good formability and is suitable for welding (Aravind et al., 2014). Figure 4 presents the designed model on the software.

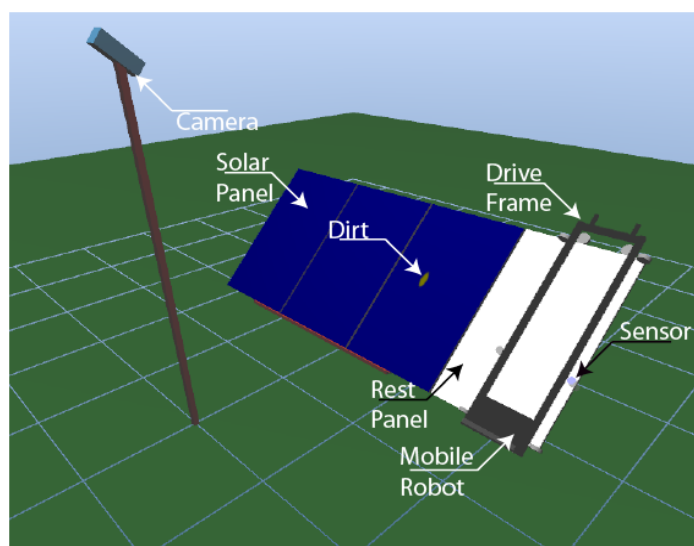

Figure 4: SimTwo Software - Robot and Solar panels.

Different models have been designed, but the twoaxis model with rails is the best solution. Allowing the robot to glide over the panel with small wheels offers less resistance, reducing the battery capacity, which significantly reduces the weight and price. A total of four position sensors are used to provide information about the position of both the frame and robot. Two of them, continuously checks if the frame reaches the edges of the solar panel and two others check for the mobile robot if it reaches the upper and lower limits.

A model was made using the FUSION 360 software, allowing a better understanding of its shape. The proposed parts, designed on this CAD software, are presented in Figure 5.

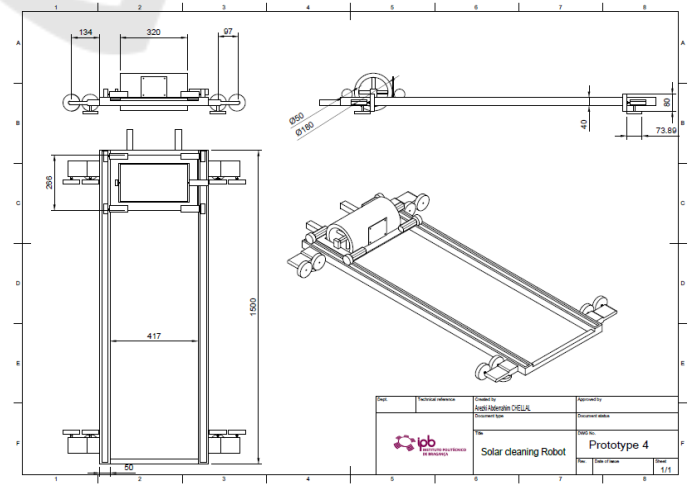

Figure 5: Robot Prototype design.

Fusion360 is a software developed by the company AUTODESK, it is a very complete software, particularly easy to use and efficient to make computer assisted drawings.

The four drive wheels must be assembled in pairs, 


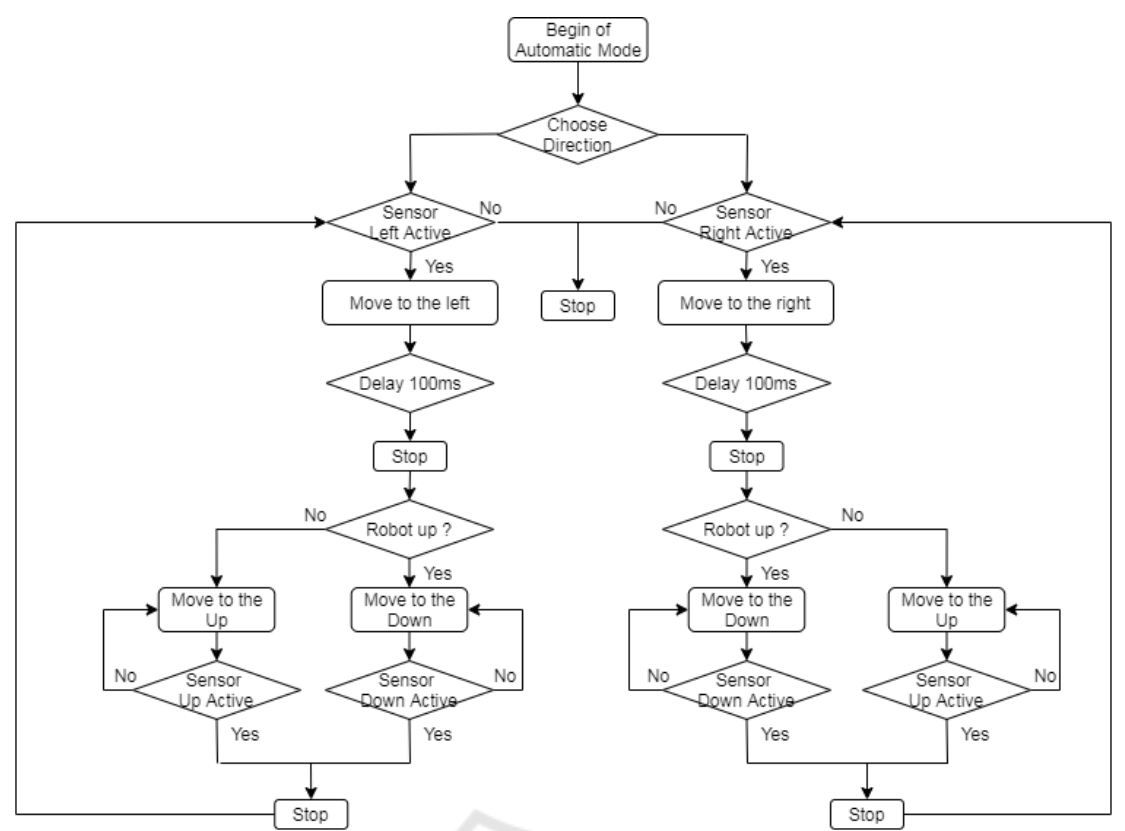

Figure 6: Automatic mode flowchart.

each pair of wheels must be connected to the other by a chain that allows only one motor to be used, which ensures that there will always be at least one drive wheel in contact with the edge when crossing the gaps between the panels. (Anderson et al., 2010).

\section{CONTROL ALGORITHMS}

The software offers the possibility of implementing the algorithm in a script in Pascal language, capable of managing the movement of the robot while performing the cleaning. It also allows to choose between three modes: manual mode, partial automatic mode and full automatic mode, as well as the direction of movement. These different modes will be detailed in the next sub-sections.

\subsection{Manual \& Partial Automatic Mode}

The manual mode is the simplest one where the robot follows the commands transmitted by pressing the corresponding button (right, left, up, down), a more in-depth discussion of it, is therefore unnecessary.

The automatic mode repeats continuously the same loop, as it can be seen in Figure 6, the robot check its position first and decide which way to move, assuming that the horizontal movement of the frame to the left is the first step of the operation. The second step of the cycle would be the robot moving vertically upwards, the $180 \mathrm{~mm}$ diameter brush is activated and moves in a circular motion using DC motors. The frame then moves to the left, towards the next cleaning area, the robot then starts the descent until it reaches the lowest point. These loops are repeated until the end of the solar panels array is reached. When the cycle is finished, the robot stops and wait for instructions.

\subsection{Fully Automated Mode}

The fully automated mode involves the analysis of the panels by a front mounted camera. The acquired image, is regularly sent with a format of 319 horizontal pixels $\times 220$ vertical pixels and is processed pixel by pixel in the developed application. The application first determines the number of panels installed, their boundaries and their respective positions, and then highlights them with a red line.

Next, the algorithm checks the RGB (red, green, blue) colour variation of each pixel included in the solar panel and when a significant amount changes, the algorithm records it as a dust particle, highlights it with a green line, identifies the position of this particle (X, Y and panel number) and assigns it a specific number (starting from 1). Then the algorithm continues to scan the rest of the panels for other dust particles or any other type of particles such as urban pollution, leaves, pollen, bird droppings. If another one is found, the algorithm records its corresponding coordinates and assigns it the next number, like a Region Labelling algorithm. Figure 7 shows the 

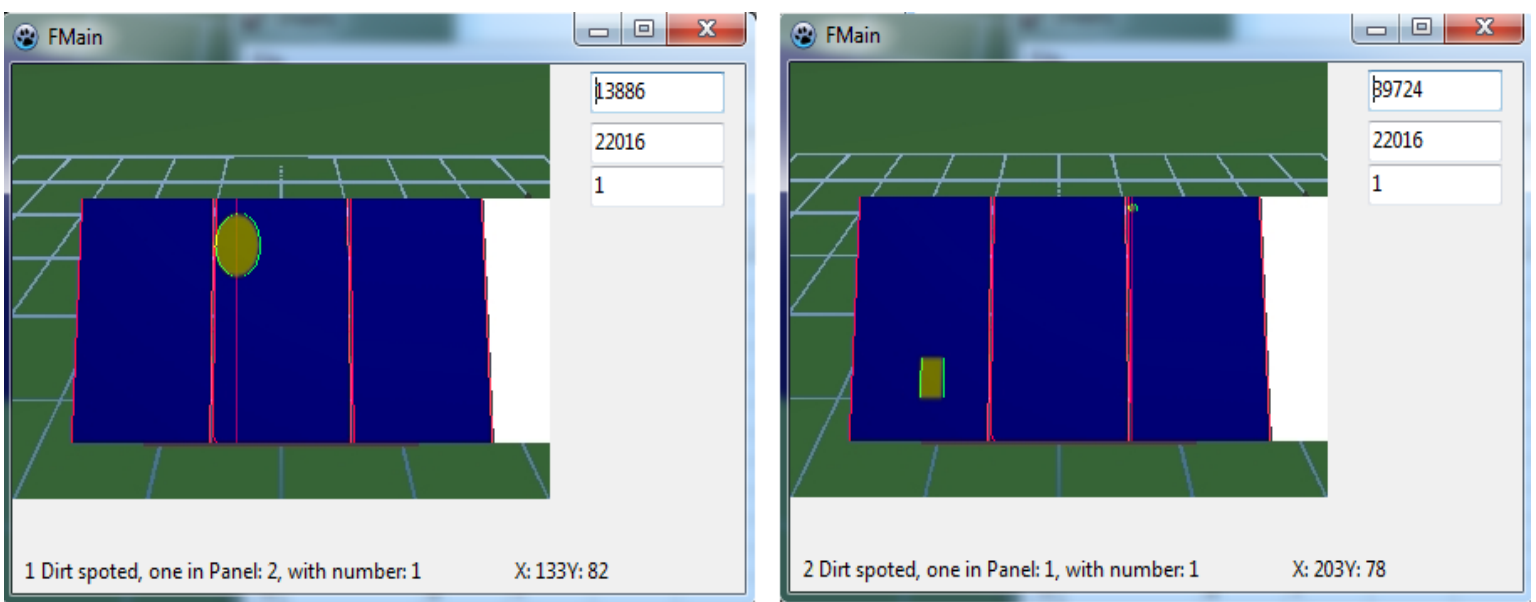

Figure 7: Application interface - Camera Picture.

application interface.

Figure 8 shows the processing algorithm flowchart implemented on the software as described previously.

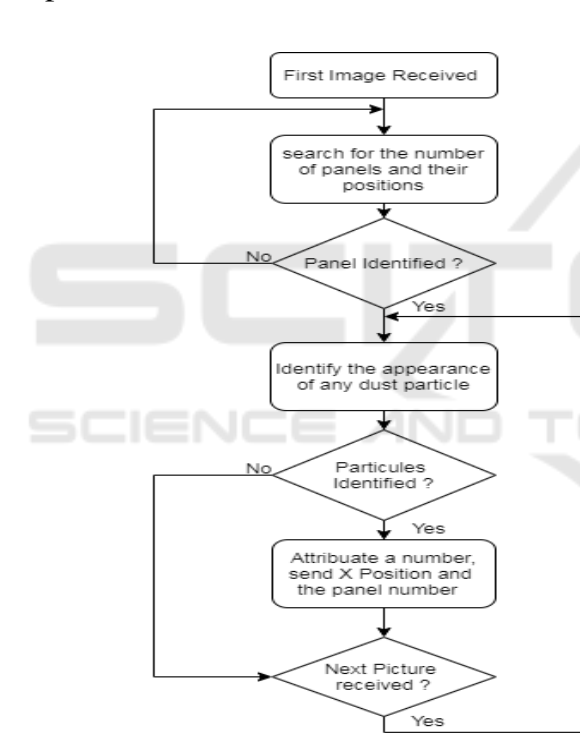

Figure 8: Picture processing flowchart.

The particle coordinates are sent to the SimTwo software and the fully automated algorithm implemented on the simulated robot moves the drive frame to the corresponding " $X$ " position and then moves the mobile robot upwards. The cleaning action is simulated using a paint tool implemented on the software that changes the colour of the dirt particles into the original background colour. As it moves, the mobile robot cleans the part of the solar panel it is passing through, changing its colour. The mobile robot then moves downwards while the driving frame moves to the resting position in the resting panel, waiting for further instructions. Figure 9 represents the fully automatic mode flowchart implemented in the software.

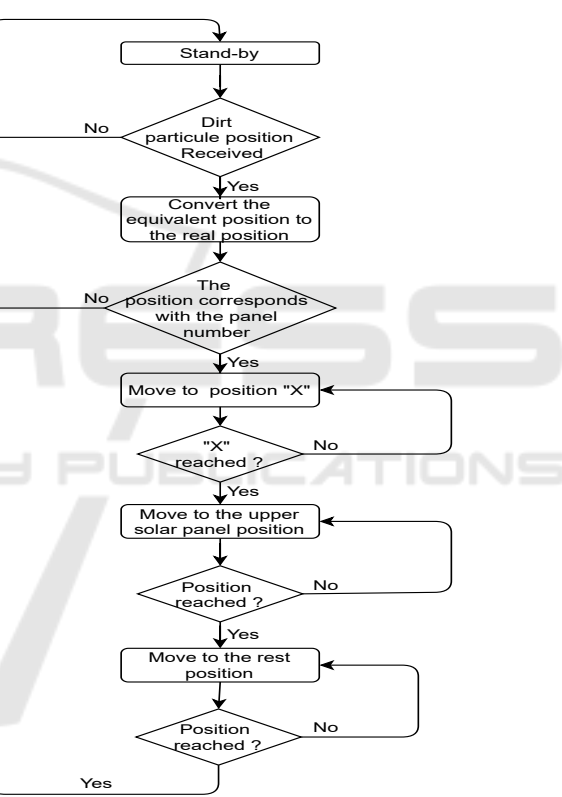

Figure 9: Fully automated mode flowchart.

It is possible to control and debug the algorithm using the "spreadsheet" window since this window is fully customisable and allows the direct display of the value of variables (sensors, motor states) and the addition of buttons (Stop and Automatic, for example). The purpose of the software part of the project is to control the simulated hardware of the system, including the mechanical components. In general, in a real case, small microcontrollers such as the well-known ATMEGA328P are preferred in such a system, as the control algorithm can easily be implemented on them (Akyazi et al., 2019). 


\section{RESULTS}

The SimTwo software has a chart plotting window which is used in this section, as it offers the possibility to plot the robot's properties at any given time (for example: position, speed, consumption and orientation). During cleaning, using the automatic mode, the frame on the panel moves half the width of the panels to the left (decreasing the X position) and stops. Sensors on both sides of the frame signal the reach of the panel's edge, at which the robot stops. The mobile robot moves up or down depending on its last position, it is made of plastic and will contain the microcontroller, battery to run the motors, electronics and will be equipped with a brush.

The proposed partial automatic mode has been tested for a large number of simulations. Figure 10 presents the graphical result of one of the simulations performed, where the reference position $(0,0,0)$ is located in the middle of the second panel, the robot starts in the rest position, located on the right with $X=1.5 \mathrm{~m}$. The blue line represents the robots movements in the "X" axis, while the red dotted line represents its position in the "Y" axis.

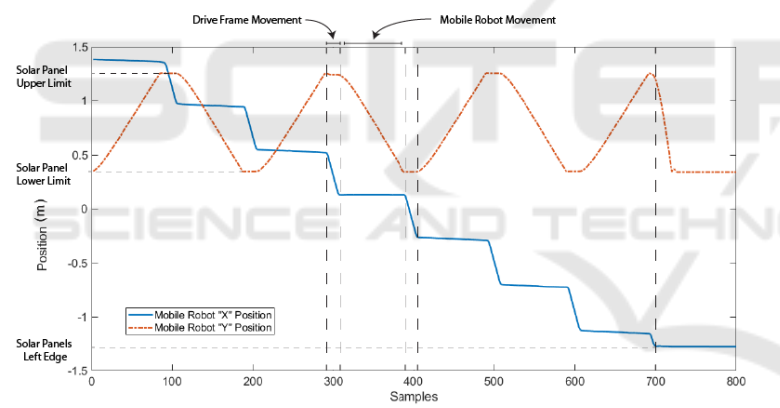

Figure 10: Mobile robot movement graphic - Automatic Mode.

The fully automated mode keeps the robot in standby while the panels are clean, as soon as a dirt particle is detected by the application, the drive frame starts to move towards the " $\mathrm{X}$ " coordinates of the dust particle, once reached the mobile robot starts its upward movement, the application is able to detect dirt particles of any shape and size, as can be seen in Figure 7.

The Figure 11 are shown the evolution of the "X" and "Y" coordinates of the mobile robot, the speed of the drive frame as it moves towards the " $\mathrm{X}$ " position and the speed of the mobile robot as it moves towards the upper part of the solar panel.

The robot movement is controlled with the use of a PID (Proportional Integral Derivative) controller taking as reference the position.

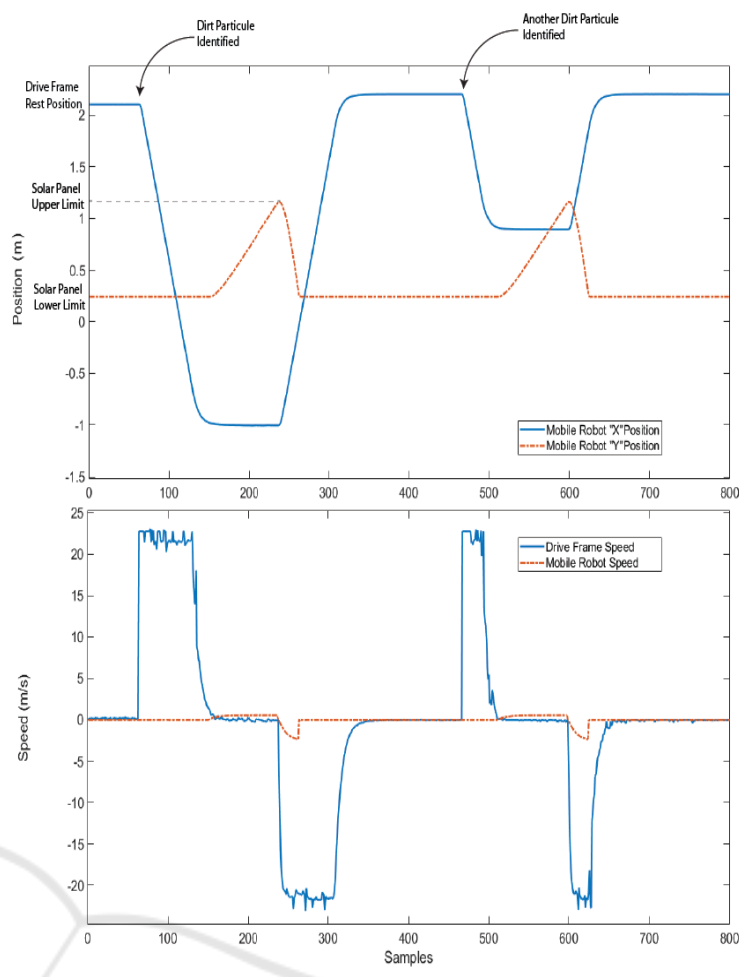

Figure 11: Mobile robot movement graphic - Fully Automated Mode.

The proposed approach based on simulation allows to validate the methodology both in image processing and robot control.

\section{CONCLUSION AND FUTURE WORK}

As the earth is in the midst of an energy transition, it is essential to be able to provide an efficiency that is, or tends to be, qualitatively equal to that of conventional electricity generation. With this proposed design, the main concerns about dust accumulation on PV panels can be effectively addressed, avoiding unnecessary losses to these panels, which can be as high as $20 \%$.

This paper proposed a mobile robot that was able to perform the assigned cleaning of dirt whatever is the panel's slope. It is important to the robot to move independently of the slope since it vary with location. Each mode developed is suitable for a specific case: the automatic mode can be used after storm or heavy soiling of the panels, while the full automatic mode is used for a day by day maintenance. The results performed on the software algorithm and on the simulated robot allowed to validate the approach.

Further development will optimize the system to 
be smaller, lighter, more user-friendly and focus on its implementation on the real hardware with the development alongside of a digital twin. The experiments with a real robot will address an RGB camera as well as a thermal camera in order to optimize the cleaning process with a sensor fusion algorithm. A docking station for the robot is also equated to charge the batteries of the robot and increase its autonomy.

\section{ACKNOWLEDGEMENTS}

This work is financed by National Funds through the Portuguese funding agency, FCT - Fundação para a Ciência e a Tecnologia within project UIDB/50014/2020.

\section{REFERENCES}

Akyazi, Ö., Şahin, E., Özsoy, T., and Algül, M. (2019). A solar panel cleaning robot design and application. Avrupa Bilim ve Teknoloji Dergisi, pages 343-348.

Anderson, M., Grandy, A., Hastie, J., Sweezey, A., Ranky, R., Mavroidis, C., and MARKOPOULOS, Y. P. (2010). Robotic device for cleaning photovoltaic panel arrays. In Mobile Robotics: Solutions and Challenges, pages 367-377. World Scientific.

Aravind, G., Vasan, G., Kumar, T. G., Balaji, R. N., and Ilango, G. S. (2014). A control strategy for an autonomous robotic vacuum cleaner for solar panels. In 2014 Texas Instruments India Educators' Conference (TIIEC), pages 53-61. IEEE.

Campana, P. E., Li, H., and Yan, J. (2013). Dynamic modelling of a pv pumping system with special consideration on water demand. Applied energy, 112:635-645.

Christian, S., Stefan, B., and Michael, K. (2015). Impact of soiling on iv-curves and efficiency of pv-modules. Solar Energy, 112:259-262.

Costa, P., Gonçalves, J., Lima, J., and Malheiros, P. (2011). Simtwo realistic simulator: A tool for the development and validation of robot software. Theory and Applications of Mathematics \& Computer Science, 1(1):17-33.

Gonçalves, J., Lima, J., Costa, P. J., and Moreira, A. P. (2013). Modeling and simulation of the emg30 geared motor with encoder resorting to simtwo: the official robot@ factory simulator. In Advances in Sustainable and Competitive Manufacturing Systems, pages 307314. Springer.

Grando, M. T., Maletz, E. R., Martins, D., Simas, H., and Simoni, R. (2019). Robots for cleaning photovoltaic panels: State of the art and future prospects. Revista Tecnología Y Ciencia, (35):137-150.

Hashim, N., Mohammed, M., Selvarajan, R. A., AlZubaidi, S., and Mohammed, S. (2019). Study on solar panel cleaning robot. In 2019 IEEE International
Conference on Automatic Control and Intelligent Systems (I2CACIS), pages 56-61. IEEE.

JKB Energy, . (2018). Get your system cleaned \& running properlys. https://www.jkbenergy.com/blog/2018/4/ 30/get-your-system-cleaned-running-properly.

Li, M., Zhang, M., Fu, Y., Guo, W., Zhong, X., Wang, X., and Chen, F. (2016). Fast and robust mapping with low-cost kinect v2 for photovoltaic panel cleaning robot. In 2016 International Conference on Advanced Robotics and Mechatronics (ICARM), pages 95-100. IEEE.

Mostefaoui, M., Ziane, A., Bouraiou, A., and Khelifi1, S. (2019). Effect of sand dust accumulation on photovoltaic performance in the saharan environment: southern algeria (adrar). Environmental Science and Pollution Research, 26(1):259-268.

Ronnaronglit, N. and Maneerat, N. (2019). A cleaning robot for solar panels. In 2019 5th International Conference on Engineering, Applied Sciences and Technology (ICEAST), pages 1-4. IEEE.

Stridh, B. (2012). Evaluation of economical benefit of cleaning of soiling and snow in pv plants at three european locations. In 2012 38th ieee photovoltaic specialists conference, pages 001448-001451. IEEE.

Sundaram, M., Prabhakaran, S., Jishnu, T., and Sharma, S. (2019). Design and analysis of an autonomous cleaning robot for large scale solar pv farms. In Proc. Int. Conf. Autom. Control, Mechtron. Ind. Eng., page 265.

Zorrilla-Casanova, J., Piliougine, M., Carretero, J., Bernaola, P., Carpena, P., Mora-López, L., and de Cardona, M. S. (2011). Analysis of dust losses in photovoltaic modules. In World Renewable Energy Congress-Sweden; 8-13 May; 2011; Sweden, number 057, pages 2985-2992. 